\title{
Plasma exchange and early thyroidectomy in thyroid storm requiring extracorporeal membrane oxygenation
}

\author{
Huilin Koh¹, Manish Kaushik², Julian Kenrick Loh ${ }^{3}$ and Chiaw Ling Chng ${ }^{1}$ \\ 1Department of Endocrinology, 2Department of Renal Medicine, Singapore General Hospital, Singapore, Singapore, \\ and ${ }^{3}$ Department of Cardiology, National Heart Centre Singapore, Singapore, Singapore
}

Correspondence should be addressed

to $\mathrm{H}$ Koh

Email

huilin.koh@mohh.com.sg

\section{Summary}

Thyroid storm with multi-organ failure limits the use of conventional treatment. A 44-year-old male presented with thyroid storm and experienced cardiovascular collapse after beta-blocker administration, with resultant fulminant multi-organ failure requiring inotropic support, mechanical ventilation, extracorporeal membrane oxygenation (ECMO) and continuous renal replacement therapy. Hepatic and renal failure precluded the use of conventional thyroid storm treatment and early plasma exchange was instituted. The patient underwent emergency thyroidectomy after four effective exchanges, with subsequent rapid reversal of multi-organ failure. The challenges of institution of plasma exchanges with ongoing ECMO support, dialysis and timing of thyroidectomy are discussed. This case highlights the important role of early therapeutic plasma exchange (TPE) as an effective salvage therapy for lowering circulating hormones and stabilization of patients in preparation for emergency thyroidectomy in patients with thyroid storm and fulminant multi-organ failure.

\section{Learning points:}

- Administration of beta-blockers in thyroid storm presenting with congestive cardiac failure may precipitate cardiovascular collapse due to inhibition of thyroid-induced hyperadrenergic compensation which maintains cardiac output.

- TPE can be an effective bridging therapy to emergency total thyroidectomy when conventional thyroid storm treatment is contraindicated.

- End-organ support using ECMO and CRRT can be combined with TPE effectively in the management of critically ill cases of thyroid storm.

- The effectiveness of plasma exchange in lowering thyroid hormones appears to wane after $44-48 \mathrm{~h}$ of therapy in this case, highlighting the importance early thyroidectomy.

\section{Background}

Thyroid storm with multi-organ failure confers very high mortality and management is challenging. When conventional treatment cannot be used, therapeutic plasma exchange (TPE) may be a salvage bridging therapy to emergency thyroidectomy. The combined use of TPE, CRRT and ECMO has not been described in the setting of thyroid storm management. This report described in detail the various treatment strategies employed in the management of a critically ill case of thyroid storm, providing guidance to physicians managing such complex cases.

\section{Case presentation}

A 44-year-old male presented with 1 week of productive cough, sore throat, rhinorrhea and breathlessness. He also had symptoms of post-tussive vomiting and 
diarrhea without abdominal pain. He was diagnosed with hyperthyroidism 4 months ago when he presented with loss of weight. Carbimazole $30 \mathrm{mg}$ daily was started by his primary physician. However, he defaulted treatment and follow-up after taking the medication for 6 weeks as his symptoms had improved. On physical examination, he was febrile at $38^{\circ} \mathrm{C}$ and was noted to be in atrial fibrillation (AF) with heart rate of $170 \mathrm{bpm}$, blood pressure of $102 / 42 \mathrm{mmHg}$, and hypoxic, with oxygen saturation of $92 \%$ on room air. He was initially alert and coherent with a Glasgow Coma Scale (GCS) of 15. He had signs of thyroid eye disease, with bilateral exophthalmos and lid retraction. There was no enlarged goiter or thyroid bruit. Cardiorespiratory examination revealed signs of congestive cardiac failure, with elevated jugular venous pressure, bilateral expiratory wheezing and bilateral pitting pedal edema up to mid shins.

\section{Investigation}

Initial laboratory investigations revealed severe hyperthyroidism, acute hepatitis, elevated inflammatory markers, cardiac enzyme and brain natriuretic peptide, as well as derangement in coagulation profile (Table 1). Electrocardiogram confirmed $\mathrm{AF}$ with rapid ventricular response of around $200 \mathrm{bpm}$. Chest radiograph revealed an enlarged heart, right patchy consolidation and bilateral pleural effusions, worse on the right. The presence of thermodysregulation, cardiac and gastrointestinal manifestations of thyrotoxicosis contributed to a high Burch-Wartofsky score of 70. A diagnosis of thyroid storm was made.

\section{Treatment}

He was promptly treated with carbimazole $30 \mathrm{mg}$, oral propranolol $20 \mathrm{mg}$ and Lugol's iodine $(130 \mathrm{mg} / \mathrm{mL})$ ten drops ( $1 \mathrm{~h}$ after carbimazole was administered). Intravenous digoxin $500 \mu \mathrm{g}$ was also initiated in view of the $\mathrm{AF}$ and cardiac failure. However, he suffered pulseless electrical activity (PEA) cardiovascular collapse during the infusion of digoxin, after propranolol was given. This was followed by three further PEA collapses, with return of spontaneous circulation each time after successful cardiopulmonary resuscitations. After a total of $40 \mathrm{~min}$ of resuscitation and subsequent return of spontaneous circulation, he was intubated and placed on inotropic support using both adrenaline and noradrenaline infusions. However, he remained hypotensive despite maximum inotropic support. Venous-arterial extracorporeal membrane oxygenation (VA-ECMO) was initiated promptly and he was immediately transferred to our institution for further treatment.

Repeat laboratory investigations revealed worsening of his liver impairment and anuric acute kidney

Table 1 Laboratory investigations on presentation of thyroid storm.

\begin{tabular}{l} 
Test \\
\hline Urea \\
Creatinine \\
Sodium \\
Potassium \\
Bicarbonate \\
fT3 \\
fT4 \\
TSH \\
Bilirubin \\
Alkaline phosphatase \\
Alanine transaminase \\
Aspartate transaminase \\
Gamma-glutamyl transferase \\
Procalcitonin \\
Lactate \\
Hemoglobin \\
Total white count \\
Platelet \\
Prothrombin time \\
Partial thromboplastin time \\
Fibrinogen \\
Brain natriuretic peptide \\
Troponin
\end{tabular}

\begin{tabular}{c}
\hline At initial presentation \\
\hline 13.3 \\
80 \\
133 \\
4.7 \\
11.3 \\
66 \\
$<0.01$ \\
133 \\
668 \\
838 \\
79 \\
0.1 \\
13.7 \\
6.7 \\
256 \\
21.3 \\
30.8 \\
3.1 \\
619 \\
60 \\
\end{tabular}

\begin{tabular}{cc}
\hline Post PEA collapse on transfer for ECMO \\
\hline 16.3 \\
249 \\
142 \\
5.1 \\
20.3 \\
23.5 \\
61.3 \\
0.266 \\
64 \\
125 \\
4021 \\
$>7000$ \\
\\
11.4 \\
8.2 \\
10.6 \\
21.1 \\
94 \\
28.7 \\
41.2 \\
0.59 \\
85
\end{tabular}

\begin{tabular}{c}
\hline Reference range \\
\hline $2.7-6.9 \mathrm{mmol} / \mathrm{L}$ \\
$54-101 \mu \mathrm{mol} / \mathrm{L}$ \\
$136-146 \mathrm{mmol} / \mathrm{L}$ \\
$3.6-5.0 \mathrm{mmol} / \mathrm{L}$ \\
$19.0-29.0 \mathrm{mmol} / \mathrm{L}$ \\
$3.2-5.3 \mathrm{pmol} / \mathrm{L}$ \\
$8.8-14.4 \mathrm{pmol} / \mathrm{L}$ \\
$0.65-3.7 \mathrm{mU} / \mathrm{L}$ \\
$7-32 \mu \mathrm{mol} / \mathrm{L}$ \\
$49-99 \mathrm{U} / \mathrm{L}$ \\
$6-66 \mathrm{U} / \mathrm{L}$ \\
$12-42 \mathrm{U} / \mathrm{L}$ \\
$14-94 \mathrm{U} / \mathrm{L}$ \\
$<0.5 \mu \mathrm{gg} / \mathrm{L}$ \\
$0.5-2.2 \mathrm{mmol} / \mathrm{L}$ \\
$14-18 \mathrm{~g} / \mathrm{dL}$ \\
$4-10 \times 10 \% / \mathrm{L}$ \\
$140-440 \times 109 / \mathrm{L}$ \\
$9.9-11.4 \mathrm{~s}$ \\
$25.7-32.9 \mathrm{~s}$ \\
$1.8-4.8 \mathrm{~g} / \mathrm{L}$ \\
$0-100 \mathrm{pg} / \mathrm{L}$ \\
$<30 \mathrm{ng} / \mathrm{L}$ \\
\end{tabular}


injury (Table 1). A transthoracic echocardiography (TTE) revealed severely impaired left ventricular systolic function with visually estimated ejection fraction of $20-25 \%$, dilatation of the right and left ventricles and cardiac congestion with pulmonary hypertension. Figure 1 summarizes the multi-organ failure and therapeutic measures initiated for this patient. A decision was made to institute early TPE until emergency total thyroidectomy could be performed. Intravenous hydrocortisone $100 \mathrm{mg} 6$ hourly and cholestyramine $4 \mathrm{~g}$ 8 hourly were also commenced as adjunctive therapy. As he was coagulopathic with disseminated intravascular coagulation (DIC), plasma exchange with $3 \mathrm{~L}$ of fresh frozen plasma (FFP) was used for the first exchange via a left femoral vein double lumen dialysis catheter. (Infomed HF440 with Granopen plasmafilter, INFOMED SA, Geneva, Switzerland.) Concurrently, VA-ECMO was ongoing via right groin angio-access with simultaneous CRRT running off the ECMO circuit (Fig. 2).

After the first TPE, his inotropic support reduced to single noradrenaline infusion. Free triiodothyronine (FT3), free thyroxine (FT4) and thyroid stimulating hormone receptor antibodies (TRAbs) also improved markedly (Fig. 3 and Table 2).

He underwent a second TPE with $3 \mathrm{~L}$ plasma exchange fluid consisting of $2 \mathrm{~L}$ of $5 \%$ human albumin and $1 \mathrm{~L}$ of FFP. Fourteen hours later, he was successfully weaned off inotropic support (Table 2). Two further plasma exchanges (3rd and 4th TPE exchange fluid: $2 \mathrm{~L}$ of $5 \%$ Albumin and $1 \mathrm{~L}$ of $\mathrm{FFP}$ ) were performed prior to the emergency thyroidectomy (Fig. 3). In view of the need for continuous intravenous heparin with the use of ECMO, a decision was made for ECMO explantation prior to thyroidectomy. He underwent successful ECMO explantation and subsequent total thyroidectomy 5 days after admission following four TPE.

\section{Outcome and follow-up}

Post-operatively, he remained off ionotropic support and was started on levothyroxine replacement, titrated to $1.8 \mu \mathrm{g} / \mathrm{kg}$. He remained intubated for another 8 days due to persistent hyperammonemia secondary to ongoing liver and renal failure, contributing to continued obtunded mental state. He was eventually extubated on post-operative day (POD) 8 when his plasma ammonia level normalized. He subsequently underwent intensive rehabilitative therapy and was discharged 9 weeks after initial presentation.

\section{Discussion}

Thyroid storm is an uncommon but life-threatening endocrine condition characterized by extreme manifestations of thyrotoxicosis. In our institution, its prevalence among hospitalized patients with thyrotoxicosis was $1.05 \%$ (1). Hyperthyroidism induces a hyperadrenergic state and its effects on the cardiovascular system are complex. In prolonged uncontrolled hyperthyroidism, a combination of relative hypervolemia, reduced myocardial contractile reserve and tachyarrhythmia can result in cardiac failure. This 'high-output' heart failure usually occurs in young individuals with severe and long-standing hyperthyroidism in the absence of any underlying heart disease (2).

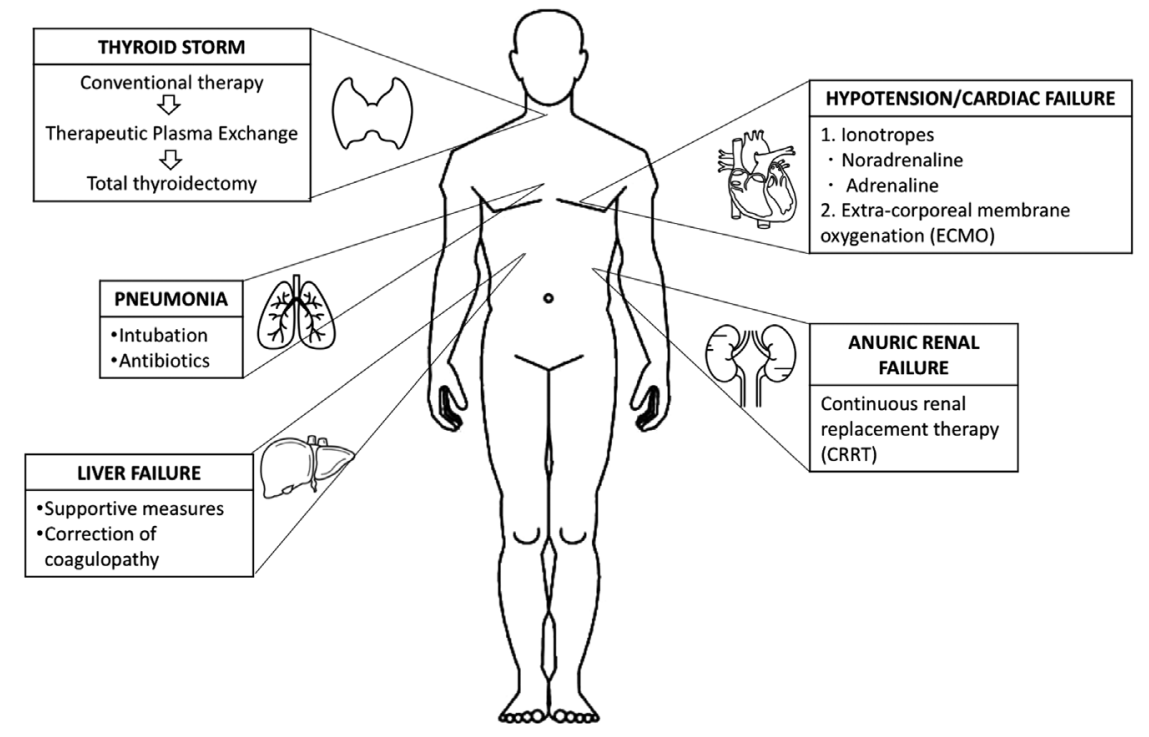

Figure 1

Schematic of multi-organ failure and therapeutic support required. 


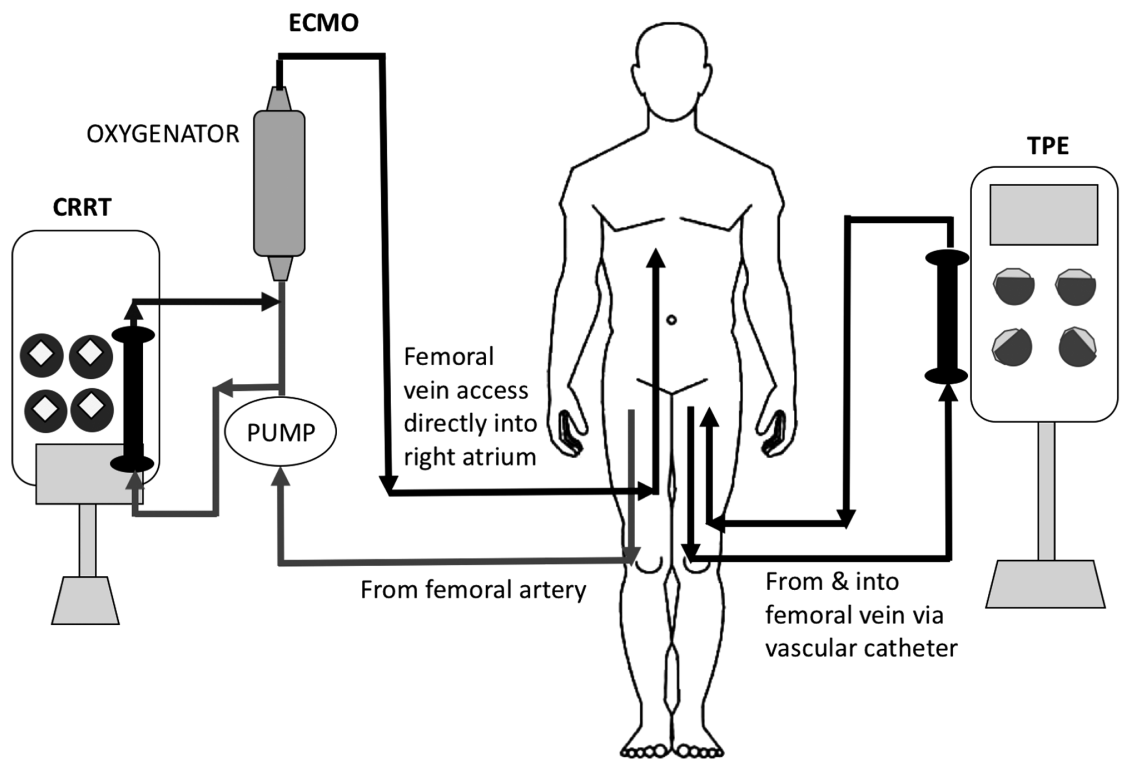

Figure 2

Schematic of simultaneous ECMO, CRRT and TPE.
Conventional thyroid storm treatment strategies simultaneously target against the synthesis, release and peripheral effects of thyroid hormones. High-dose propranolol is commonly employed to counter the hyperadrenergic state of thyrotoxicosis, with the added benefit of inhibiting peripheral conversion of $\mathrm{T} 4$ to the more potent T3. However, our patient experienced severe cardiovascular collapse after propranolol was administered. A systematic literature review by Abubakar et al. (3) found nine published reports of beta-blocker-induced cardiovascular collapse. Notably, low cardiac output heart failure with suppressed left ventricular ejection fracture (LVEF) was a common finding in these reports. While propranolol can counteract a high-output failure in the context of a preserved ejection fraction, it is potentially detrimental to administer it in 'low-output' cardiac failures with depressed ejection fraction, due to the negative inotropic effects of the beta-blockers in acute decompensated cardiac failure. Our patient had clinical evidence of heart failure on presentation and was subsequently found to have low ejection fraction of $20-25 \%$ on TTE. Although digoxin was initiated in our patient, he collapsed before the loading dose was completed. It is also noteworthy that cardiac glycosides are less effective in the hyperthyroid compared to euthyroid state due to its altered metabolism in hyperthyroidism (4). Thus, this case highlights the potential life-threatening adverse effect of using propranolol in patients with thyroid storm who present with heart failure.

TPE is generally employed in the management of thyroid storm when conventional treatment fails or cannot be used due to toxicity. Although rare, the use of TPE in thyroid storm is not new. It is now a category III indication for thyroid storm (i.e. optimum role of apheresis therapy is not established; decision making should be individualized) according to the American Society for Apheresis (ASFA) 2013 guidelines on use of therapeutic apheresis (5). However, our case is the first reported use of TPE together with ECMO and CRRT in the treatment of thyroid storm. A search of the literature found that concurrent use of ECMO and TPE has been reported to be safe in adults and children in non-thyroid storm settings (6). The largest case series by Dyer et al., which reviewed 76 cases which utilized both TPE and ECMO noted the most common indications for initiating TPE on top of ECMO were multi-system organ failure and transplant rejection (6). During TPE, a patient's plasma is extracted from its cellular components and replaced with a colloid such as albumin and/or plasma. Thyroid hormone-

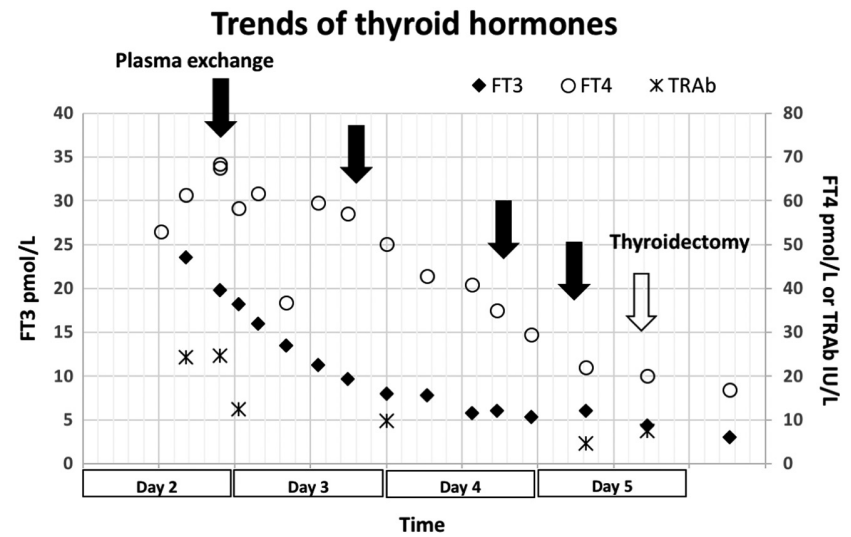

Figure 3

Trend of thyroid hormones and thyrotropin receptor antibodies with plasma exchange. 
Table 2 Serial FT3, FT4 and TRAb measures following four cycles of therapeutic plasma exchange.

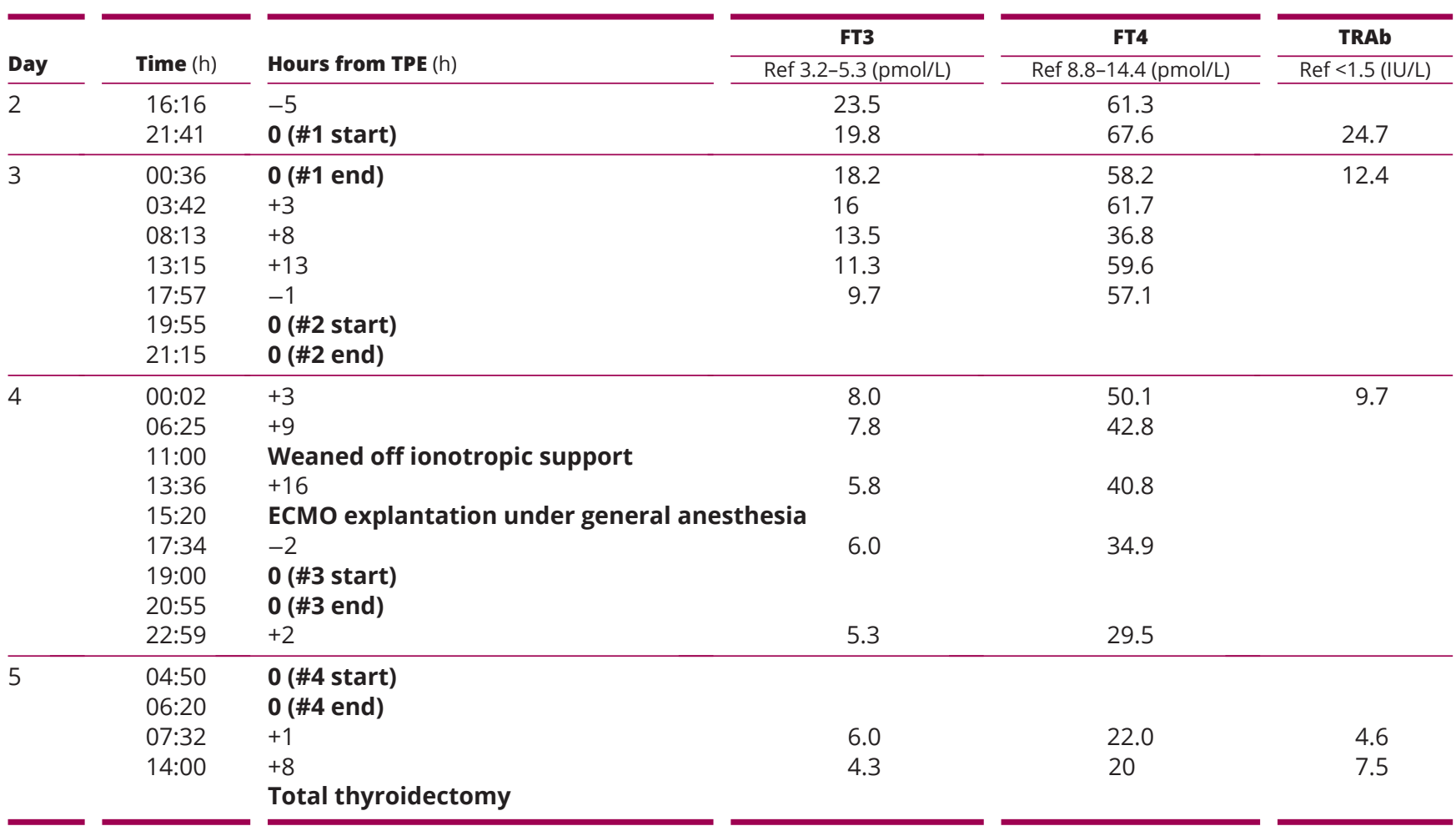

binding globulins (TBGs) with bound thyroid hormone is removed from circulation and the replacement colloid provides new binding sites for circulating free hormones, thereby reducing free hormone concentrations. TPE also removes circulating antibodies (TRAb), overall ameliorating thyrotoxicosis. However, clotting factors and immunoglobulins are also removed with TPE, increasing the risk of bleeding and infections. With the concomitant use of intravenous heparin to prevent clotting of the extracorporeal therapy circulations, the risk of bleeding increases manifold. Hence, we recommend that a portion of the TPE replacement fluid should consist of plasma (FFP), in order to replace some of these depleted clotting factors. However, this needs to be balanced with avoiding clotting of the ECMO and CRRT circuits; therefore, the remaining portion of TPE replacement fluid is made out of human albumin. The patient's blood-activated clotting time (ACT) was monitored and heparin infusion was titrated to a target ACT of 180-200s (institution ECMO protocol), and the patient was closely monitored for signs of bleeding. In addition, albumin was used together with FFP in the exchange colloid to reduce the volume of plasma required, as well as reduce the complications of FFP transfusion such as citrate toxicity and hypocalcemia.

This case also highlights the importance of the early use of TPE in thyroid storm. TPE was initiated within
$24 \mathrm{~h}$ of presentation when it was clear that hepatic and renal failure precluded the continued use of conventional thyroid storm treatment. Several authors $(7,8)$ as well as the ASFA guidelines (5) recommend to conduct TPE as early as possible in thyroid storm. Binimelis et al. described a 30-fold decrease in total thyroxine levels with plasma exchange as compared to conventional treatment, and this effect was proportional to the serum level of thyroxine (7). This suggests that TPE is more efficient if done early and also highlights the narrow effective therapeutic window of this treatment. Rapid clinical improvement was apparent in our patient after the first two plasma exchanges, although serum FT3 and FT4 remained high. We observed in our patient that the efficacy of plasma exchange dropped after the third TPE (44-48 $\mathrm{h}$ after initiation of TPE).

The aim of TPE as a salvage bridging therapy was to render the patient fit enough for total thyroidectomy (definitive treatment of thyroid storm) under general anesthesia. Table 3 highlights the parameters we monitored to determine fitness for operation. There are differing opinions with regards to the timing of surgery after the last TPE. Ezer et al. reported safety with thyroidectomy within $24 \mathrm{~h}$ after last exchange; unusual bleeding at the operation site only occurred in one out of eleven surgeries (9). Ozbey et al., however, reported increased bleeding compared to 
Table 3 Parameters monitored while awaiting total thyroidectomy.

\begin{tabular}{l}
\hline Parameter \\
\hline Thyroid hormone, fT3 \\
Blood pressure \\
Cardiac ejection fraction \\
Coagulopathy profile \\
PT/PTT
\end{tabular}

\section{Target}

Normal

Unsupported/minimal ionotropic support

Wean off ECMO

Normal

the usual with thyroidectomy performed within $24 \mathrm{~h}$ of TPE, in two out of four patients (10). They recommend delaying thyroidectomy to $48 \mathrm{~h}$ after the last TPE and also to use FFP in the replacement fluid for the plasma exchange right before operation, to reduce the risk of bleeding. Our patient underwent thyroidectomy within $8 \mathrm{~h}$ of TPE without any bleeding complications. Perioperatively, he received plasma, cryoprecipitate and platelet transfusion for correction of coagulopathy. We suggest that thyroidectomy is safe within $24 \mathrm{~h}$ of TPE as long as any coagulopathy is promptly treated with blood products.

\section{Conclusion}

We highlight the first case of concurrent ECMO, TPE and CRRT in the treatment of thyroid storm. Early use of TPE was beneficial with rapid clinical improvement observed. The number of exchanges as well as timing of surgery with respect to last exchange needs to be considered on a case by case basis.

\section{Declaration of interest}

The authors declare that there is no conflict of interest that could be perceived as prejudicing the impartiality of this case report.

\section{Funding}

This work did not receive any specific grant from any funding agency in the public, commercial or not-for-profit sector.

\section{Patient consent}

Written informed consent has been obtained from the patient for publication of the submitted article and accompanying images.

\section{Author contribution statement}

$\mathrm{H} \mathrm{K}$ was responsible for patient management, writing and editing the manuscript. $M K, J K L$ were involved in patient management, editing and final

\section{Rationale}

Reduce the hyperadrenergic state caused by thyrotoxicosis Reduce general anesthetic (GA) risk

Reduce surgical and GA risk

IV heparin infusion with ECMO use increases peri-operative bleeding risk

Reduce peri-operative bleeding risk

approval of the manuscript. C L C was involved in the patient management, overall supervision, editing and final approval of the manuscript.

\section{References}

1 Swee du S, Chng CL \& Lim A. Clinical characteristics and outcome of thyroid storm: a case series and review of neuropsychiatric derangements in thyrotoxicosis. Endocrine Practice 201521 182-189. (https://doi.org/10.4158/EP14023.OR)

2 Klein I \& Danzi S. Thyroid disease and the heart. Circulation 2007116 1725-1735. (https://doi.org/10.1161/ CIRCULATIONAHA.106.678326)

3 Abubakar H, Singh V, Arora A \& Alsunaid S. Propranolol-induced circulatory collapse in a patient with thyroid crisis and underlying thyrocardiac disease: a word of caution. Journal of Investigative Medicine High Impact Case Reports 20175232470961774790 . (https:// doi.org/10.1177/2324709617747903)

4 Huffman DH, Klaassen CD \& Hartman CR. Digoxin in hyperthyroidism. Clinical Pharmacology and Therapeutics 197722 533-538. (https://doi.org/10.1002/cpt1977225part1533)

5 Szczepiorkowski ZM, Winters JL, Bandarenko N, Kim HC, Linenberger ML, Marques MB, Sarode R, Schwartz J, Weinstein R, Shaz BH, et al. Guidelines on the use of therapeutic apheresis in clinical practice-evidence-based approach from the apheresis applications committee of the American Society for Apheresis. Journal of Clinical Apheresis 201025 83-177. (https://doi. org/10.1002/jca.20240)

6 Dyer M, Neal MD, Rollins-Raval MA \& Raval JS. Simultaneous extracorporeal membrane oxygenation and therapeutic plasma exchange procedures are tolerable in both pediatric and adult patients. Transfusion 201454 1158-1165. (https://doi.org/10.1111/trf.12418)

7 Binimelis J, Bassas L, Marruecos L, Rodriguez J, Domingo MLP, Madoz P, Armengol S, Mangues MA \& de Leiva A. Massive thyroxine intoxication: evaluation of plasma extraction. Intensive Care Medicine 198713 33-38. (https://doi.org/10.1007/BF00263555)

8 Muller C, Perrin P, Faller B, Richter S \& Chantrel F. Role of plasma exchange in the thyroid storm. Therapeutic Apheresis and Dialysis 201115 522-531. (https://doi.org/10.1111/j.17449987.2011.01003.x)

9 Ezer A, Caliskan K, Parlakgumus A, Belli S, Kozanoglu I \& Yildirim S. Preoperative therapeutic plasma exchange in patients with thyrotoxicosis. Journal of Clinical Apheresis 200924 111-114. (https:// doi.org/10.1002/jca.20200)

10 Ozbey N, Kalayoglu-Besisik S, Gul N, Bozbora A, Sencer E \& Molvalilar S. Therapeutic plasmapheresis in patients with severe hyperthyroidism in whom antithyroid drugs are contraindicated. International Journal of Clinical Practice 200458 554-558. (https://doi. org/10.1111/j.1368-5031.2004.00140.x)

Received in final form 28 May 2019

Accepted 2 July 2019 